\title{
Interaction between oxidative stress and diabetes: a mini-review
}

\begin{abstract}
The Diabetes Mellitus (DM) is increasing in incidence and prevalence worldwide, taking the shape of the pandemic proportions, which is expected to increase further over a couple of decades. In 2019, an estimated $9.3 \%$ of the population (463 million people), globally had DM. Projection of future DM burden may increase to $10.2 \%$ (578 million people) by 2030 and $10.9 \%$ (700 million people) by next 25 years (2045). The decrease in immunity in diabetic patients has been a significant concern for physicians and patients alike. The role of mitochondria and it is cellular signaling can elaborate on this problem in depth. Mitochondria is a rich source of reactive oxygen species (ROS) and plays a crucial role in cellular metabolism for energy. They are targets of ROS and many different cellular signals like mitochondrial membrane apoptosis due to hyperglycemia and ROS. The interplay between oxidative stress and diabetes further activated, leading to increased permeability of mitochondrial membrane \& apoptosis; this leads to a decrease in immunity in diabetic patients leading to increased mortality and morbidity. Hyperglycemia and ROS major pathways of apoptosis through mitochondrial activation in diabetic patients leading to decreased immunity. They can become drug targets for further intervention of this problem in Diabetic patients.
\end{abstract}

Keywords: mitochondria, oxidative stress, diabetes mellitus, apoptosis, ROS
Volume 7 Issue 2 - 2020

\author{
Ashish Ashish, ' Arunim Shah, ${ }^{2}$ Shubhrendu \\ Shekhar Pandey, ${ }^{3}$ Royana Singh' \\ 'Department of Anatomy, Institute of Medical Science Banaras \\ Hindu University, India \\ ${ }^{2}$ Stem Cell Research Center, Department of Hematology, Sanjay \\ Gandhi Postgraduate Institute of Medical Sciences, India \\ ${ }^{3}$ Department of Orthopaedics, Institute of Medical Sciences \\ Banaras Hindu University, India
}

Correspondence: Royana Singh, MD, PhD, Department of Anatomy, Institute of Medical Science Banaras Hindu University, Varanasi, 221005, Uttar Pradesh, India, Email royaasingh@bhu.ac.in

Received: April II, 2020 | Published: June 29, 2020
Abbreviations: DM, diabetes mellitus; ROS, reactive oxygen species; IR, insulin resistance; GLUT4, glucose transporter 4; T2DM, type 2 diabetes mellitus; OS, oxidative stress; GPX, glutathione peroxidase; MAMs, mitochondrial-associated membranes; CAT, catalase; SOD, superoxide dismutase; ATP, adenosine triphosphate; ETC, electron transport system; MMPTP, mitochondrial membrane permeability transition pore; mtROS, mitochondrial ROS

\section{Diabetes}

The tremendous and sharp worldwide increase of patients suffering from Diabetes Mellitus (DM) over the last few decades is probably one of the leading health concerns. ${ }^{1}$ Diabetes mellitus, popularly known as diabetes, is a metabolic, endocrine disorder ${ }^{2}$ characterized by increased blood sugar levels over an extended period. Diabetes occurs due to a defect in insulin action, secretion, insulin response, or both. The chronic hyperglycemia and Insulin resistance (IR) of diabetes type 2 leads to damage or dysfunction of critical organs like coronary artery disease, or renal dysfunction, blood vessels, stroke, heart, kidneys, nerves, and eyes. ${ }^{3}$ Its association with retinopathy, neuropathy, and nephropathy is also frequently established. ${ }^{4}$ Lifestyle, sedentary living, and high energy dietary intake have not even exempted young children from developing T2DM at an unexpectedly early age. ${ }^{5}$ A large number of women in developed countries are affected by diabetes compared to men. ${ }^{6}$ Insulin-stimulated glucose uptake is facilitated by GLUT4 (glucose transporter 4) primarily found in adipose tissues and striated muscles such as skeletal and cardiac muscles. ${ }^{7}$ Disruption in glucose uptake is a sign of the onset of T2DM. ${ }^{8}$ Innate and adaptive immunity are found to be functionally compromised during the progression of T2DM. Mitochondria is the center of cell metabolism disturbance and plays a significant role in maintains the Redox balance. Redox balance and maintenance of ROS levels crucially regulates disease development and progression. ${ }^{9}$
Oxidative stress-related disease leads to structural and metabolic impairment activating the apoptotic pathway of mitochondria, redox homeostasis resulting in cellular death and alteration of Adaptive immunity and decrease in ATP production in Diabetes Mellitus.?

In this review, we are elaborating Molecular events linking, Oxidative Stress, and mitochondria, which lead to Mitochondrial Dysfunction and Apoptosis mitochondrial membrane potential other cells of adaptive immunity in Diabetes Mellitus. The inhibitory effects in mitochondria alteration of Adaptive immunity and oxidative stress lead to inflammatory responses activating the production of various major pathways and inflammatory markers such as various cytokines regulated by transcriptional mediated oxidative metabolic stress, are mentioned and discussed.

\section{Epidemiology}

With the corona COVID 19 pandemic at its peak in the world in the 21 st century, type 2 diabetes may be the second biggest epidemic in history. The global prevalence of diabetes Mellitus is devastating. ${ }^{10}$ With many factors attributing to its cause like genetic, environment lifestyle, behavioural and at present shifted to intrauterine and epigenetics changes diabetes mellitus is approaching pandemic proportions. ${ }^{11}$ In 2019 , an estimated $9.3 \%$ of the world population (463 million people), globally had DM, which may increase to $10.2 \%$ (578 million people) by 2030 and $10.9 \%$ (700 million people) by next 25 years (2045). ${ }^{12}$ Diabetes is a problem of higher economic countries and half of those suffering are not aware of having diabetes. The most significant growth in prevalence has been found in high-income countries $(10.4 \%)$ than in low-income countries $(4.0 \%)$ and higher in urban $(10.8 \%)$ than rural $(7.2 \%)$ area. The prevalence of Diabetes in the United States of America has just doubled from 11.9 million in 2000 to 21.9 million in 2014, and 31 million in $2019 .{ }^{13}$ However, in recent times, the global proportion of people with DM has risen 
significantly in India (77 million) and China (116 million) besides the United States ( 31 million). ${ }^{12}$ As the rise continues, the prevalence of DM patterns will increase by 2045, with 147, 134 and 37 million people in India, China and Pakistan, respectively, in the near future. ${ }^{12}$

\section{Oxidative stress}

Oxidative stress (OS) is an essential consequence of life in aerobic environments. Excess of free radicals and reduced antioxidants leads to oxidative stress in the cells. ${ }^{14}$ Imbalances antioxidants' capacity to scavenge free radicals, including superoxide anion $(\mathrm{O} 2 \bullet-)$, peroxynitrite $(\mathrm{ONOO}-)$, and hydroxyl radical $(\bullet \mathrm{OH}){ }^{15}$

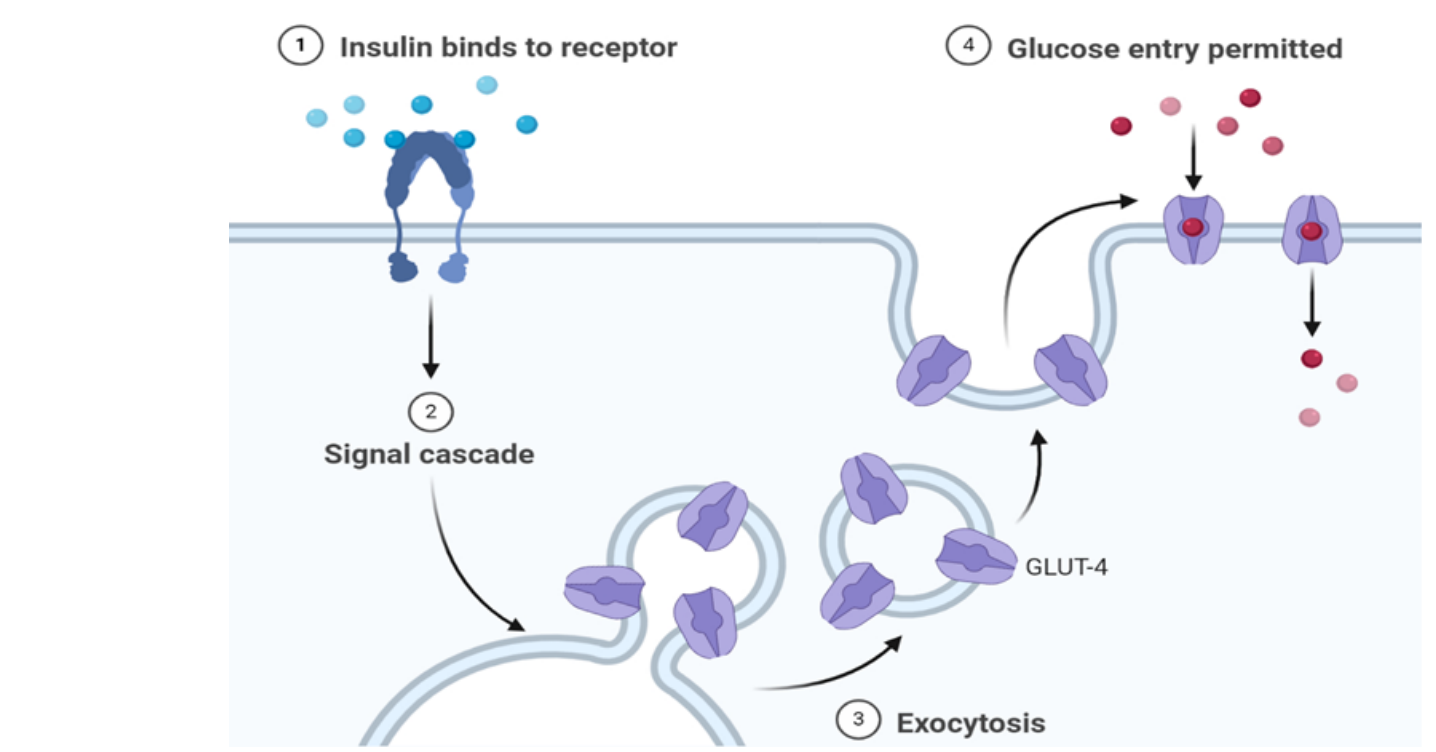

Various enzyme are involved in scavenging free radicals such as catalase(CAT), superoxide dismutase (SOD), Glutathione Peroxidase (GPX), glutathione reductase etc. disruption in the internal defense mechanism leads to oxidative damage of lipids, proteins, and nucleic acids. As a consequence, cellular cytotoxicity and genotoxicity increase, which may lead to carcinogenesis during cell proliferation of mutated cells. ${ }^{16}$ Oxidative stress damages mitochondrial-associated membranes (MAMs), mitochondrial ROS promotes Endoplasmic reticulum stress. ${ }^{17}$ The glucose transporter GLUT4 facilitates insulinstimulated glucose uptake into muscle and adipose tissue. Defects in glucose uptake represent an early step in the development of type 2 diabetes mellitus Figure 1.

Figure I Activation of the glucose transporter GLUT4 by insulin.

The interplay between oxidative stress interactions diabetes mellitus

Although many vital aspects of the etiology of DM are still unclear. The mitochondria serve a pivotal role in energy transduction, signaling, and cell death pathways related to diabetes mellitus. ${ }^{18}$ Perturbations in Structural and functional mitochondria affect the cause and complications of diabetes. ${ }^{19}$ Growing evidence suggests that free radicals have an essential effect on the pathophysiology of diabetes progression and complications. Hyperglycemia is associated with a gradual rise in oxidative stress. ${ }^{20}$ Oxidative stress is considered crosstalk between both mechanisms is related to alterations in glucose homeostasis and can lead to the diabetes-associated insulin-resistance status plays a vital role in the onset and change of this condition. ${ }^{21}$

\section{Mitochondria: role in apoptosis and necrosis}

Mitochondria are considered the power generators of the cell, converting oxygen and nutrients into adenosine triphosphate (ATP). ${ }^{17}$ The membrane potential of mitochondria is disrupted, causing cellular injury as a result of various pathological conditions. The leading cause of cellular injury is complicated than anticipated. ${ }^{22}$ Mitochondrial membrane hyperpolarization and morphological changes are essential in inflammatory cell activation..$^{23}$ Membrane permeability transition in mitochondria is enhanced by calcium, ischemia/reperfusion, oxidant chemicals, and other agents affecting the coronary arteries' heart, kidneys, nerves, and other tissues. ${ }^{24}$ This event leads to mitochondrial and cellular swelling, ATP depletion, ion deregulation, and activation of derivative enzymes, plasma membrane failure, and cell lysis. There by triggering events leading to necrotic cell death. ${ }^{25}$ The mitochondrial membrane permeability is also involved in apoptotic cell death. ${ }^{26}$ Cell death and the role of membrane permeability transition is to release proapoptotic proteins from the mitochondria into the cytosol, utilizing cellular ATP to activate the apoptotic cascade. ${ }^{27}$ Thus mitochondrial membrane permeability induces both necrotic and apoptotic death in mitochondrial cell dysfunction. ${ }^{28}$

\section{Role of reactive oxygen species (ROS) in apoptosis and necrosis pathway in diabetes}

Although it is very well known that hyperglycemia has several detrimental effects, that understanding of the fundamental relationship between abnormal high glucose and cell death metabolism has only been clarified recently. ${ }^{29}$ ROS regulates several necrotic and apoptotic pathways in diabetes. ${ }^{30}$ Oxidative stress is triggered by a nutritional imbalance. As a result, the antioxidant defense is hampered, and the Pro-oxidant burden is increased. ${ }^{31}$ A high level of ROS damages the mitochondrial function and disrupts the electron transport chain (ETC) ${ }^{32}$ The mitochondrial membrane potential is a target of ROS under pathologic conditions. Correctly, mitochondrial membrane permeability transition pore (MMPTP) opens, causing the release of cytochrome $\mathrm{C}$ from the mitochondrial matrix to the cytoplasm, which activates pro-apoptotic caspases. ${ }^{33}$ Mitochondrial potential disruption causes oxidative stress and inflammation, amplified oxidative burst, and propagate inflammation further. ${ }^{34}$ Superoxide anions are 
produced by mitochondria as a by-product from the mitochondrial respiratory chain complexes I and III as electron leakage. These Superoxide anions and its product are also termed as mitochondrial ROS (mtROS). ${ }^{35}$ Through mitochondrial leakage, increased mtROS production and mitochondrial disruption increases the expression of inflammatory cytokines leading to enhanced responsiveness of cells to inflammatory signaling and activate inflammasome. ${ }^{36}$ Figure 2 Mitochondria are one of the primary sources of ROS/RNS and also mediate cellular apoptosis and cell death. Type 2 diabetes gives rise to hyperglycemia which causes changes in Oxidative stress leading to changes in the mitochondrial membrane Potential that could be related to altered mitochondrial dynamics, which is associated with $\mathrm{T}$ cell dysfunction and apoptosis. There is evidence that mitochondrialtargeted antioxidants and mitochondrial function are decreased in type 2 diabetes leading to Cell Death and apoptosis of monocyte and lymphocyte cells.

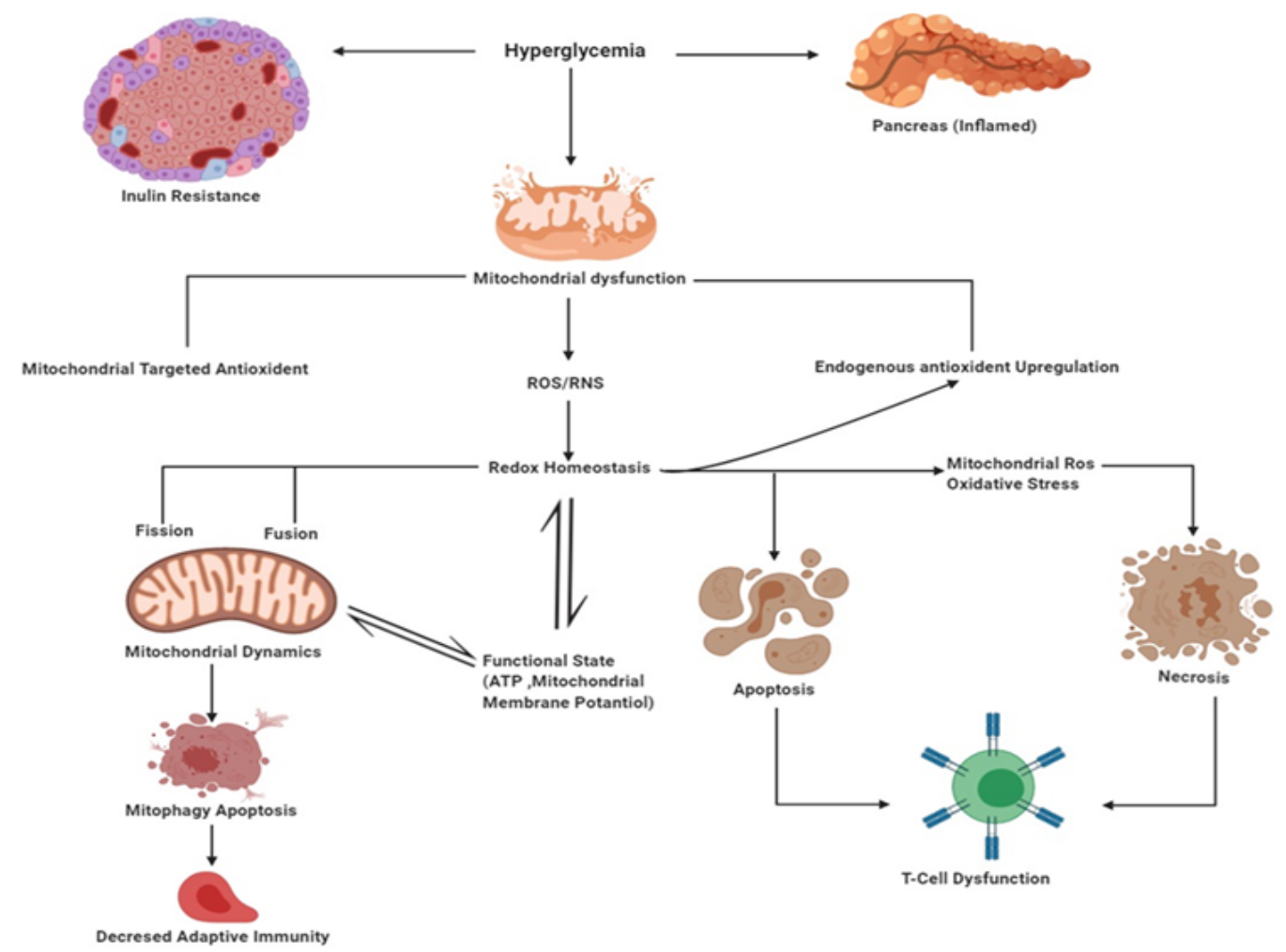

Figure 2 Interaction between mitochondrial dynamics, oxidative stress, and mitochondrial function in type 2 diabetes.

\section{Mitochondrial dysfunction}

Reduced mitochondrial biogenesis, mitochondrial number, altered membrane potential, and decreased activities of oxidative proteins due to the accumulation of ROS in cells and tissues is a condition of mitochondrial dysfunction. ${ }^{37}$ Mitochondrial membrane dysfunction is represented by mitochondrial membrane potential, morphological changes, mass, functional losses of the mitochondria. ${ }^{38}$ And structural disruption such as fragmentation of mitochondria and inflammation are observed, leading to increased mitochondrial fission under pathologic conditions. ${ }^{39}$

\section{Conclusion}

Apoptosis is crucial for the maintenance of tolerance and the prevention of autoimmunity. Diabetes can effectively increase oxidative stress leading to mitochondrial dysfunction and increased permeability of mitochondrial membranes. Activation of both these pathways leads to increased mitochondrial permeability and other changes leading to apoptosis in Cells of immunity and T cells. In diabetic patients, decreased immunity leads to mortality and morbidity in a large number of patients. This is an of the further research area and various pharmacological agents blocking and modulating and different pathways should be investigated for their effect on stabilizing effect on mitochondria in $\mathrm{T}$ cells and Immunity in general.

\section{Acknowledgments}

This research was Sponsored by Multi-Disciplinary Research Units (MRUs) a grant by ICMR-Department of Health Research.

\section{Conflicts of interest}

The authors declared that there are no conflicts of interest.

\section{Funding}

None.

\section{References}

1. Kaveeshwar SA, Cornwall J. The current state of diabetes mellitus in India. Australas Med J. 2014;7(1):45-48.

2. Salmon AB. Oxidative stress in the etiology of age-associated decline in glucose metabolism. Longev Heal. 2012;1(1):7.

3. American Diabetes Association AD. Diagnosis and classification of diabetes mellitus. Diabetes Care. 2009;33 Suppl 1(Suppl 1):S62-S69. 
4. Sami W, Ansari T, Butt NS, et al. Effect of diet on type 2 diabetes mellitus: A review. Int J Health Sci (Qassim). 2017;11(2):65-71.

5. Rhee EJ. Diabetes in Asians. Endocrinol Metab (Seoul). 2015;30(3):263269.

6. Bjerregaard LG, Jensen BW, Ängquist L, et al. Change in overweight from childhood to early adulthood and risk of type 2 diabetes. $N$ Engl J Med. 2018;378(14):1302-1312.

7. Rowley WR, Bezold C, Arikan Y, et al. Diabetes 2030: insights from yesterday, today, and future trends. Popul Health Manag. 2017;20(1):612 .

8. Leto D, Saltiel AR. Regulation of glucose transport by insulin: traffic control of GLUT4. Nat Rev Mol Cell Biol. 201;13(6)383-396.

9. Osellame LD, Blacker TS, Duchen MR. Cellular and molecular mechanisms of mitochondrial function. Best Pract Res Clin Endocrinol Metab. 2012;26(6)711-723.

10. Zimmet PZ. Diabetes and its drivers: the largest epidemic in human history? Clinical Diabetes and Endocrinology. 2017;3:1

11. Popkin BM, Adair LS, Ng SW. Global nutrition transition and the pandemic of obesity in developing countries. Nutr Rev. 2012;70(1):3-21.

12. Saeedi P, Petersohn I, Salpea P, et al. Global and regional diabetes prevalence estimates for 2019 and projections for 2030 and 2045: results from the international diabetes federation diabetes atlas, 9th edition. Diabetes Res Clin Pract. 2019;157:107843.

13. Lin J, Thompson TJ, Cheng YJ, et al. Projection of the future diabetes burden in the United States through 2060. Popul Health Metr. 2018;16(1):9.

14. Asmat U, Abad K, Ismail K. Diabetes mellitus and oxidative stress-a concise review. Saudi Pharm J. 2016;24(5):547-553.

15. Lobo V, Patil A, Phatak A, et al. Free radicals, antioxidants and functional foods: impact on human health. Pharmacogn Rev. 2010;4(8):118-126.

16. Kurutas EB. The importance of antioxidants which play the role in cellular response against oxidative/nitrosative stress: current state. Nutrition Journal. 2016;15:71.

17. Burgos-Morón E, Abad-Jiménez A, de Marañón AM, et al. Relationship between oxidative stress, ER stress, and inflammation in type 2 diabetes: the battle continues. J Clin Med. 2019;8(9):1385.

18. Ceriello A. Oxidative stress and glycemic regulation. Metabolism: Clinical and Experimental. 2000;49(2 Suppl 1):27-29.

19. Yan L-J. Pathogenesis of chronic hyperglycemia: from reductive stress to oxidative stress. J Diabetes Res. 2014:137919.

20. Bhansali S, Bhansali A, Walia R, et al. Alterations in mitochondrial oxidative stress and mitophagy in subjects with prediabetes and type 2 diabetes mellitus. Front Endocrinol (Lausanne). 2017;8:347.

21. GZ R, AS C, RH S, et al. Relationship of oxidative stress as a link between diabetes mellitus and major depressive disorder. Oxid Med Cell Longev. 2019:8637970
22. Kalogeris T, Baines CP, Krenz M, et al. Cell biology of ischemia/ reperfusion injury. Int Rev Cell Mol Biol. 2012;298:229-317.

23. Ceriello A, Motz E. Is oxidative stress the pathogenic mechanism underlying insulin resistance, diabetes, and cardiovascular disease? the common soil hypothesis revisited. Arterioscler Thromb Vasc Biol. 2004;24(5):816-823.

24. Lemasters JJ, Theruvath TP, Zhong Z, et al. Mitochondrial calcium and the permeability transition in cell death. Biochim Biophys Acta. 2009; 1787(11):1395-401.

25. Nieminen A-L. Apoptosis and necrosis in health and disease: role of mitochondria. Int Rev Cytol. 2003;224:29-55.

26. Tsujimoto Y, Shimizu S. Role of the mitochondrial membrane permeability transition in cell death. Apoptosis. 2007;12(5):835-840.

27. Wang C, Youle RJ. The role of mitochondria in apoptosis. Annu Rev Genet. 2009;43:95-118.

28. Kang J, Pervaiz S. Mitochondria: redox metabolism and dysfunction. Biochem Res Int. 2012:896751.

29. Stehouwer C DA. Microvascular dysfunction and hyperglycemia: a vicious cycle with widespread consequences. Diabetes. 2018; 67(9):1729-1741.

30. Volpe CMO, Villar-Delfino PH, dos Anjos PMF, et al. Cellular death, reactive oxygen species (ROS) and diabetic complications. Cell Death Dis. 2018;9(2):119.

31. Kim SH, Kim H. Inhibitory effect of astaxanthin on oxidative stress-induced mitochondrial dysfunction-a mini-review. Nutrients. 2018;10(9):1137.

32. Hahn A, Zuryn S. Mitochondrial genome (mtDNA) mutations that generate reactive oxygen species. Antioxidants (Basel). 2019; 8(9):392.

33. Akopova OV, Kolchynskayia LY, Nosar' VY, et al. The effect of permeability transition pore opening on reactive oxygen species production in rat brain mitochondria. Ukr Biokhim Zh. 1999;83(6):46-55.

34. Salminen A, Ojala J, Kaarniranta K, et al. Mitochondrial dysfunction and oxidative stress activate inflammasomes: impact on the aging process and age-related diseases. Cell Mol Life Sci. 2012;69(18):2999-3013.

35. Cui H, Kong Y, Zhang H. Oxidative stress, mitochondrial dysfunction, and aging. J Signal Transduct. 2012:646354.

36. Chen Y, Zhou Z, Min W. Mitochondria, oxidative stress and innate immunity. Front Physiol. 2018;9:1487.

37. Marchi S, Giorgi C, Suski JM, et al. Mitochondria-ros crosstalk in the control of cell death and aging. J Signal Transduct. 2012:329635.

38. Grazioli S, Pugin J. Mitochondrial damage-associated molecular patterns: from inflammatory signaling to human diseases. Front Immunol. 2018;9:832.

39. Galloway CA, Yoon Y. Mitochondrial morphology in metabolic diseases. Antioxid Redox Signal. 2013;19(4):415-430. 